\title{
SCTP-WSN New Extension for More Reliable Sparse Wireless Sensor Networks
}

\author{
To Be Applied in the Area of Data Collection Systems for the Control and the Surveillance \\ of Drinking Water Supply Systems
}

\author{
${ }^{1}$ S.Sadouni, ${ }^{2}$ M.Benslama, ${ }^{3}$ A.Mebarki \\ ${ }^{12}$ Electromagnetism and telecommunication laboratory \\ ${ }^{2}$ Laboratories of Territory Sciences, Natural Ressources and \\ Territory Management (LASTERNE) \\ University of Constantine \\ Constantine, Algeria \\ ${ }^{1}$ salaheddinesadouni@umc.edu.dz \\ ${ }^{2}$ ma_benslama@yahoo.fr \\ 33.-mebarki@yahoo.com
}

\begin{abstract}
Wireless sensor network applications are becoming the main concern of several studies. Not only they allow to maintain more varied measures in several fields notably that of real time hydraulic systems control and surveillance. But their dense deployment decreases granularity which can lead to the comprehension of some unexplainable phenomena. However, the effectiveness of a sensor network not only depends on the quality of the sensors individually, but also on their collaboration. The latter is only possible with reliable routing protocols which have to adjust to fit the constraints imposed by the application. With this aim in mind, we suggest in the current work a new extension of SCTP (Stream Control Transmission Protocol) that we call SCTP-WSN (wireless sensor network). This extension will have as a main function to improve the communicative performances of sparse wireless sensor networks with dynamic topologies. In order to do this, this extension will have to improve the availability of the links in sparse sensor networks by a better integration of the Multihoming concept, representing therefore a more reliable data collection subsystem for a real time detection of possible disorders affecting drinking water supply systems.
\end{abstract}

Keywords-Wireless Sensor Network; SCTP; AODV; Multihoming; drinking water supply syste

\section{INTRODUCTION}

Recent advances in the fields of wireless communication technologies and micro-electronics reduced the size of sensors, their cost and the diversification of their types. The latter integrate a sensing unit that is responsible for measuring physical quantities in its close environment and then to transform them into numeric ones. They also integrate a processing and data storing unit, as well as a wireless transmission module. Therefore, the deployment of these entities that allows measured data collection and transmission into collection points forms a wireless sensor network [1].

\author{
A.Beylot \\ Institut de Recherche en Informatique de Toulouse \\ University of Toulouse \\ Toulouse, France \\ andre-luc.beylot@enseeiht.fr
}

Wireless sensor networks have multiple applications in various fields such as: the military, the environmental, the domestic, and the medical fields, as well as the surveillance of critical infrastructures such as water systems [2].

The functioning of a sensor network could be compared to that of an Ad hoc network, since a sensing node has generally weak calculating, power and communication saving capacities. Furthermore, the sensors are responsible for data study and routing into the collection point named sink. The latter recovers data with the help of different sensors and transmits them to the treatment center. In order to do so, wireless sensor networks borrow Ad hoc routing protocols [3]. On top of broadcasting, we distinguish three types of Ad hoc routing protocols: Proactive, reactive and hybrid protocols. In this article, we shed the light on the category of reactive routing, more precisely on Ad Hoc On demand Distance Vector Routing (AODV).

The major advantage of a wireless sensor network is its capacity to move quickly even in difficult conditions. Whereas, most of the applications are based upon dynamic and uncontrollable topologies in which nodes continually and unexpectedly change their positions. This continual instability of the network causes recurrent failures of the data transfer routes between the different sensor and sink nodes. Consequently, the intermediary nodes will have to delete the data packets since they do not have an alternative route. To recover the transmission, the broadcasting sensor node (source) will have to launch a new route discovery procedure. This results in diminishing the reliability of the whole network [2].

For such reasons, we suggest in this article a new extension of SCTP protocol. We call it: SCTP-WSN. Its main function would be to define the best method of integrating Multihoming to WSN This should be done while taking into account its characteristics. The previously mentioned concept will focus on the creation of multiple paths between sensors and sinks, and ensuring the transparent passage of a broken-down node to one that works. In order to validate the contribution of this new extension to network performances in this article, we will be 
based upon a comparative study of the average throughput between SCTP-WSN application and the standard SCTP version in different WSN topologies. This could be done by using Riverbed Modeler software which represents a new version of Openet Modeler.

With this aim in mind, section two (2) will tackle real time control and surveillance of hydraulic infrastructure of drinking water supply. On the other hand, section three (3) will be devoted to tackle AODV routing protocol, as well as SCTP transport protocol and the integration of the Multihoming concept. The functioning of SCTP-WSN new extension will be presented in section four (4). As far as section five (5) is concerned, we will present the simulation results obtained by the simulator Riverbed Modeler. Finally, section (6) will allow us to conclude and suggest a new perspective.

\section{REAL TIME CONTROL AND SURVEILLANCE OF DRINKING WATER SUPPLY SYSTEMS}

The protection of public health requires that drinking water supply systems meet very strict quality and security criteria. It is therefore necessary to prevent any intrusion in hydraulic systems. It is also very important to immediately detect any contamination whether it be intentional or accidental [4]. The protection of visible resources could be done with the help of old means of physical detection and perimeter protection. However, as far as important hydraulic systems are concerned such as dams, it is more difficult to implement such protection.

With this aim in mind, it is essential to create a global anomaly detection system that relies on permanent water quality surveillance so as to optimize the reaction time, and to implement the necessary adequate corrective measures [4]. In order to do so, the realization of this system applies the following elements:

\section{A. Quantitative and qualitative data collection subsystem}

Constructed of a network of wireless sensors that are spread over the water surface to be controlled and mentored. In the current paper, we will be focusing on the implantation of our new extension in this subsystem.

\section{B. Dynamic subsystem}

Which takes into charge the calculation of the optimal topographical positioning of the different sensor nodes and sinks that constitute the network according to the needs and the meteorological criteria

\section{Collected data presentation software}

It also takes in charge the detection and the notification of abnormal situations.

\section{AODV ROUTING PROTOCOLS}

AODV is a distant vector protocol. It essentially represents an enhancement of DSDV algorithm (Destination-Sequenced Distance Vector). The objective of AODV protocol is to provide a service which is completely based on the principle of the path on demand, because it never demands a path unless it needs it. The nodes do not maintain routing data, and they do not exchange periodically their routing table. Besides, a node has to establish a connection request before discovering or maintaining any routes. Discovery packets are only transmitted when necessary. AODV allows distinguishing the management of local connections from that of the general topology. It also permits broadcasting the changes of local connections with mobile nodes which could be in need of this information [5].

AODV is based upon the use of two mechanisms:

- Route discovery between source and destination nodes (sensor and sink nodes) via the use of Route REQuest messages (RREQ) and Route REPly (RREP).

- Route maintenance, via the transmission of Route ERRor messages (RERR). This mechanism anticipates the local reparation of a route. It also saves the transmission of routing messages.

AODV protocols create and maintain routes based on the principle of broadcasting which causes in some cases important delays before a route is open [5].

\section{SCTP TRANSPORT PROTOCOL AND MULTIHOMING CONCEPT}

SCTP protocol was designed to remedy certain TCP protocol limitations related to IP phone signaling (like the problem of head of line blocking). It provides a reliable transport service of messages coming from users' applications.

It is connection oriented. An SCTP connection is called 'association'. It is possible to multiply several message streams within the same association (Multistreaming service). This shows in the possibility of transporting multiple message signals within the same SCTP packet. The messages are condensed in SCTP/IP packets [6].

In addition, SCTP enables the launching of Multihoming by introducing the possibility of associating multiple IP addresses to the same SCTP port. Then, several paths are available to link two remote SCTP links. At some time, there is only one active path to be used to transport data. The other paths are to be used as a backup plan in case the active path becomes unavailable.

\section{A. The Concept of Multihoming Characteristics and Applications}

Multihoming refers to the possibility of an internet protocol to use multiple IP addresses for the same exchange. The latter's main function involves the localization of a machine in the network. This situation takes place when a node has multiple communication interfaces, or because the network where it is localized is connected to internet via multiple routers or via a multi-cellular router. The main applications of Multihoming are:

- Speeding the network changes by obtaining anticipated addresses.

- Sharing the load by dividing the stream on multiple paths.

- Enhancing the network's performances by switching data stream from an unavailable node to an available one. In the coming lines, we will focus on the 
application of Multihoming in order to increase the connectivity of sparse WSN [7].

The integration of Multihoming could be achieved by the NAT mechanism (Network Address Translation). However, the most widely used solution at the moment is SCTP protocol. Multihoming, which is regarded as an essential property of this protocol, permits an SCTP association to be connected with multiple source and destination addresses. Thus every mobile device could be reached via multiple IP addresses. SCTP only brings a path saving mechanism. There is just one active path at once. Sharing the load does not belong to the current characteristics of the protocol. Each node could be accessible by different transport addresses that are fixed just when the association is open. Transport addresses (IP address lists+ SCTP port) are exchanged while the initialization phase of an SCTP association takes place. Thus transmissions toward multihomed nodes could gain more robustness against network failures or congestion problems by realizing a transparent passage between an unavailable or a congested path and another available one, without any connection failure, because SCTP considers IP addresses of a Multihomed client as reachable 'different paths' [7].

\section{CONSTRAINTS RELATED TO SCTP AND AODV USE WITH WSN}

Several research projects have revealed that the integration of Multihoming to MANET networks significantly enhances their performances. This shows in the ability to realize a transparent passage between an unavailable link and an available one. The same applies to mobile sensor networks, increasing their communicative performances requires multiplying the possible paths between sensor nodes and sink nodes. Nevertheless, there is much to be said about the constraints related to these networks notably their limited power source and their instable topologies.

The use of SCTP transport protocol; which takes into charge Multihoming; combined with AODV routing protocol seems to be the most adequate in order to multiply the available routes between sensor and sink nodes. Hence, there are two possible scenarios that arise:

- AODV is based upon a broadcasting mechanims for the implantation of a route between sensor and sink nodes. This process proves to be very expensive in terms of network resources and power intake. As a result, this causes important delays before the route is open. These delays often surpass the average delays that are usually permitted by software for an optimal coverage of the work zone.

- AODV is a unipath protocol. When it is on demand, it takes in charge the setting up of one route. This functioning policy enforces the intermediary nodes that are situated between mobile sensors and the Sink to delete data packets in the case of route failure.

- Since the main role of SCTP protocol is to take into charge the integration of Multihoming, this protocol could dispose of alternative routes on the level of transport layer. However, if the primary path fails,
AODV will not be aware of the existence of a secondary one.

In order to remedy the constraints stated above, a new extension of SCTP protocol; called SCTP-WSN; should be suggested. The latter will have as a function to enhance the communicative performances of WSN via the integration of Multihoming. In addition, it will anticipate the possible changes of the WSN topologies, optimize power intake, and reduce reply delays.

\section{SCTP-WSN NEW EXTENSION FUNCTIONING}

In order to enchance WSN communicative performances, our new extension SCTP-WSN has to take into consideration two main elements:

- The functioning terms of SCTP-MANET extension base as exposed in former research projects.

- Achieving hybrid centralized and decentralized network managing architectures

As its name suggests, SCTP-MANET is supposed to increase the robustness of MANET networks via a fast failing routes recovery. As far as this humble work is concerned, we will very briefly expose the two main elements that are necessary for the functioning of SCTP-MANET [8]

- Cross layer interface.

- Sequential in-order-of-importance route creation.

\section{A. Cross Layer Interface}

As far as the cross layer interface between two network layers is concerned, it will be inspired by the model of interaction type into an intermediary entity. The intermediary cross layer interface will be situated between two transport layers (the third and the fourth ones). Such configuration will allow SCTP-MAN in case of primary route failure to automatically shift the packets transmission to a secondary route and launch a primary route discovery procedure.

The data to be saved and shared by this interface is: RREQ, RREP and RRER messages of the AODV protocol, as well as the messages establishing an association of the SCTP protocol: INIT and INIT-ACK. The implantation of SnCLI (Sensor cross layer interfaces) and SkCLI (Sink cross layer interfaces) will be done respectively at the level of sensor and sink nodes.

\section{$B$. The sequential in-order-of-importance route creation}

To discover routes, AODV protocol uses a broadcasting process. Hence, SCTP-WSN numbers its routing messages (RREQ and RREP) in order to create in-order-of-importancesequenced routes (One primary route and two secondary ones).

The RREP and RREQ numbering is done in binary code from 1 to 3 . These numbers will be implanted in the fields RESERVED. The latter are successively composed of 9 bits to 0 for RREP messages and 11 bits to 0 for RREQ messages. These fields are ignored once received.

It is possible for multiple routes to request the same node at once. If the latter becomes inactive, all of the former routes will fail. That is why by the time a discovery route operation is 
launched, the numbered RREQ message ignores the nodes that are numbered differently so as to create disjointed routes.

As far as transport is concerned, SCTP-WSN uses the same numbering principle for INIT and INIT-ACK messages. That is to say in binary code from 1 to 3 . These numbers will be implanted in binary at the level of flag fields of INIT and INITACK. Composed of 8 bits to 0 , the flag fields have no particular function to accomplish.

\section{Achieving hybrid Centralized and Decentralized Network Managing Architectures}

The absence of WSN centralized infrastructures make these networks more vulnerable especially when it comes to their dynamic topologies and their power intake. A WSN sensor network refers to a collection of mobile sensors which are interconnected by a wireless technology that shape together a temporary network without the help of any centralized administration or any fixed support. Thus, after the necessary data collection of the study area is done, it is directly sent to a centralized node (or to multiple nodes) in other words (sink).

For the purpose of countering the effects of these two constrains, as well as improving the WSN communicative performances; the current paper suggests the use of a hybrid architecture where the network has most of the time a decentralized management and centralized one in certain cases. Since the sink node plays a major part in this hybrid architecture, two scenarios may rise:

In the first scenario, WSN network operates normally. Therefore, the Sink only collects the data that is sent by the sensors and forwards it to the final user.

However, in the second scenario the Sink is actively involved in the routing data since all sensor nodes have to communicate with the sink at some point. In addition to the collected data, these sensors send other useful data in order to optimize the routing process.

As far as our paper is concerned, much focus will be devoted to tackle:

- Direct neighborhood with a sensor node.

- The list of the nodes which constitute its primary route to the sink, as well as its two secondary routes.

Establishing a hybrid management to the WSN implies providing a global view of the network at the level of Sink. A sensor node which is incapable of building a secondary route to the Sink sends it a particular message. As a reply, the Sink forwards the list of the neighboring nodes along with the one of their secondary routes. This action will allow the sensor nodes to optimize their power intake, and prevent data loss which usually results from abrupt link failures.

\section{Establishing an SCTP-WSN Association}

The establishement of an SCTP-WSN association allows each sensor node to communicate with the Sink via three different routes: one primary route, and two secondary ones. By using the concept of Multihoming in the case of primary route failure, SCTP-WSN allows a sensor to automatically and transparently forward its data to a second or a third secondary route.

At the beginning of the association, every sensor seeks a primary route in order to join the Sink. In order to do so, this sensor uses the same SCTP-MANET association establishement steps [8]:

- The transmission of INIT messages via SCNCLI interface which asks AODV protocol to define a route to the Sink.

- AODV sends a RREQ1 and waits for a reply from the Sink. This REEEQ1 will be saved in SkCLI which replies with a RREP1.

- Once REEP1 gets to SnCLI of the damanding sensor, it replies with an INIT1 through the route that is established by RREP1. When this INIT1 attains the Sink, it replies with an INIT-ACK1.

- Afterwards, the same SCTP association establishment steps take place along with the transmission of the messages INIT, INIT-ACK, COOKIE-ECHO and the route will be considered as primary.

- Regarding the secondary routes, SCTP-WSN resumes the steps from 1 to 3 , but using INIT2 for the first secondary route, and INIT3 for the second secondary route.

- After the establishment of each route (primary and secondary), the sensor nodes must compulsorily send them to the Sink along with the list of their direct neighbors.

- However, it is sometimes impossible for a sensor to define two other secondary routes. Hence, it is necessary to shift to a centralized WSN management by involving the Sink.

- After ten unsuccessfull attempts to establish secondary routes by the sensor, the latter sends a specific INIT4 message via its SnCLIto the Sink through the primary route.

- With the reception of the INIT4 message by the SkCLI of the Sink, it saves it and replies with the transmission of the primary and secondary routes of the current demanding node neighbors.

- Once this data is received by the sensor, it attempts to establish a first secondary route and it alerts (notifies) the Sink.

Like the second secondary route, the sensor resumes the same steps from 8 to 10 by using INIT5. The SCTP-WSN takes in charge the integration of secondary route IPs to the list of active routes.

\section{SimULATION}

\section{A. Simulation Software}

For the purpose of highlighting the contribution of our new SCTP-WSN extension, we have to realize a comparative study between multiple scenarios. With this aim in mind, we use the 
Riverbed Modeler Academic Edition 17.5 software. The latter is a simulator that allows to model a network functioning during the conception phase. Therefore, it enables the reproduction of the structure of a WSN network and its components. It also permits the collection of lots of statistic data out from the execution of different scenarios belonging to this study [9].

\section{B. Performance Criteria}

As far as performance criteria are concerned, we suggest in this study the average throughput which is received by the Sink. In other words: the average rate of use (throughput) which shows in the relationship between the information quality transmitted by the average throughput (per second) and the network flow.

\section{Simulation Scenarios}

In the current paper, we are intending to conduct a comparative study between two different scenarios. The latter will use AODV as a routing protocol. This protocol already exists in the simulation software. However, regarding data transport, the first scenario will use SCTP protocol and the second one will use our new SCTP-WSN extension. These two protocols i.e. SCTP and SCTP-WSN do not exist in the simulation software. Hence, we have to implant them in their basic versions. This implies the fact that a number of modifications will have to take place at the level of general automatons that are used in the transport layer.

\section{The first scenario}

As already mentioned, the transport layer must use SCTP protocol. The latter is not yet implanted in the simulation tool. Therefore, we have to create it within the basis of this simulator. In order to do so, we have added four (4) particular states that characterize SCTP protocol namely INIT, INIT$\mathrm{ACK}, \mathrm{COOKIE-ECHO}$ and COOKIE-ACK. The running of each state of the latter is programmed in advance in $\mathrm{C}++$ language. Lastly, all sensor nodes as well as Sink must implant SCTP protocol.

\section{E. The Second Scenario}

Regarding the second scenario, in the transport layer we program SCTP-WSN extension functioning at the level of process model. That is why we add two new states (Find-new) and (Find-after) to the SCTP functioning. The (Find-new) state will have as a function to define the second and the third routes after the activation of the SCTP-WSN association takes place. On the other hand, (Find-after) state begins searching for secondary routes after ten unsuccessful attempts of (Find-new). We must also add two new processors: SnCLI at the level of each sensor and SkCLI at the level of Sink. Situated between the transport and routing layers, SnCLI and SkCLI will play the part of cross layer interface between the layer 3 and 4 . In addition, they will also have to take into charge the messages that are numbered INIT, INIT-ACK RREP and RREQ.

Both scenarios SCTP and SCTP-WSN are applied on four different WSN network topologies:

- 1. The first network contains 10 nodes.

- 2. The second network contains 20 nodes.
- 3. The third network contains 30 nodes.

- 4. The Last one contains 40 nodes.

The WSN coverage zone stretches over 16 square kilometers. The simulation time could reach an hour. On each sensor, we activate 4 wireless interfaces with a different IP address in order to set up the concept of Multihoming (Table1).

TABle I. The Simulation PARAMETERs USED FOR BOth SCENARIOS SCTP AND SCTP-WSN.

\begin{tabular}{|l|l|}
\hline Receive buffer (bytes) & 65535 \\
\hline Send buffer size & 71680 \\
\hline Maximum (INIT1, COOKIE) ACK delay (sec) SCTP-WSN & 0.2 \\
\hline Maximum (INIT2, INIT 3) ACK delay (sec) SCTP-WSN & 0.4 \\
\hline Maximum (INIT4, INIT 5) ACK delay (sec) SCTP-WSN & 2.0 \\
\hline Maximum (INIT, COOKIE) ACK delay (sec) SCTP & 0.2 \\
\hline Maximum ACK Segments & 2 \\
\hline Duplicate ACK Threshold & 3 \\
\hline Receive buffer (bytes) & 65535 \\
\hline Send buffer size & 71680 \\
\hline
\end{tabular}

VIII. THE RESULTS

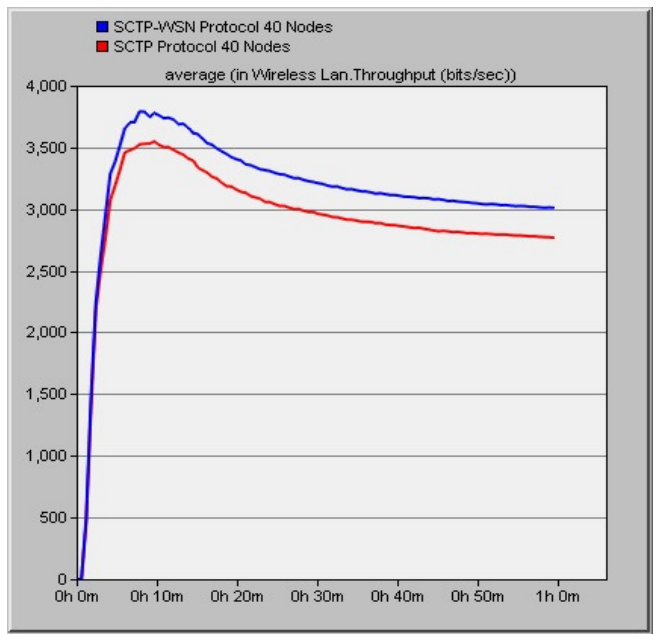

Figure 1. Forty-node WSN Average Throughput

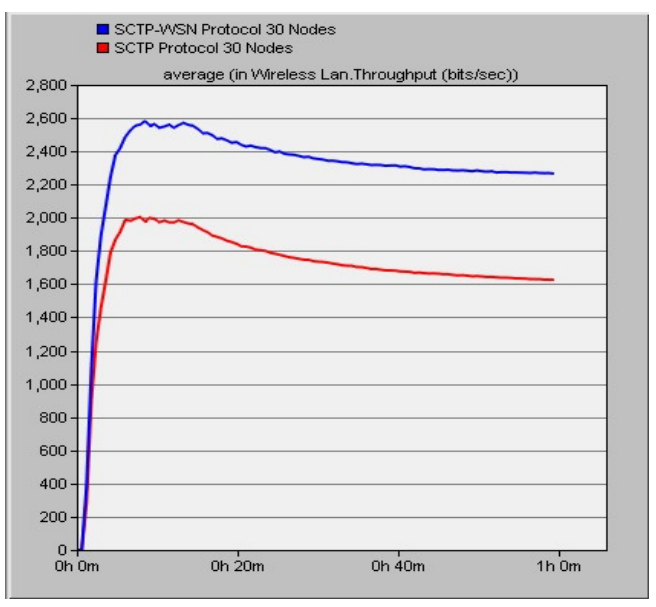

Figure 2. Thirty-node WSN Average Throughput 


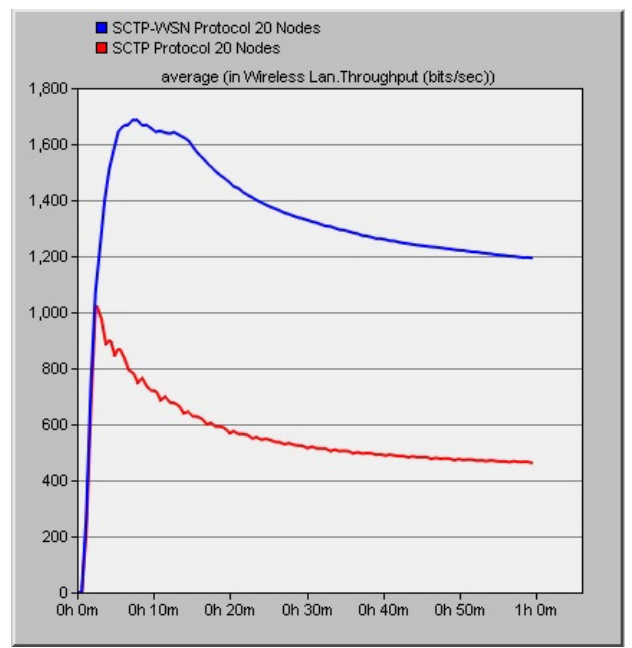

Fig. 3. Twenty-node WSN Average Throughput

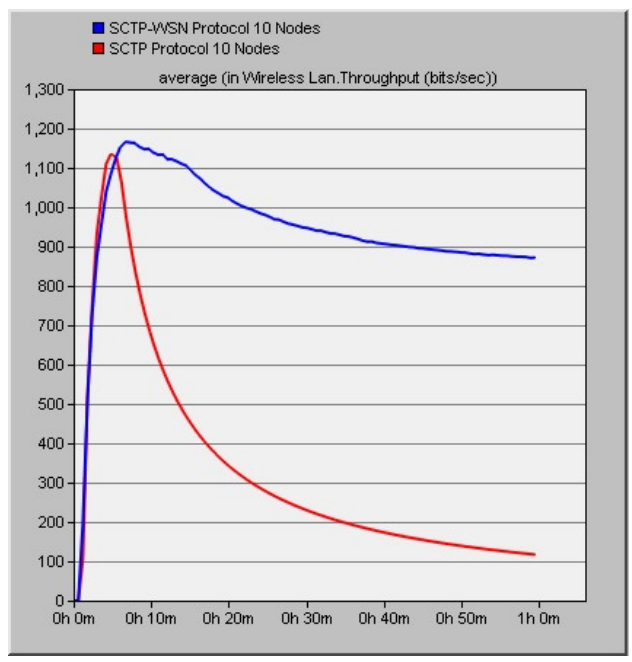

Fig. 4. Ten-node WSN Average Throughput

Fig. 1, 2, 3 and 4 demonstrate the existence of a gap in the average throughput between both scenarios. This gap increases significantly as long as the number of the WSN sensors decreases.

\section{A. Results Discussion}

Within the densest wireless sensor networks, the data transport routes availability is considered to be extremely crucial. Hence, a sensor which loses its connection with the Sink is able to immediately restore it. In return, the sparse WSN involves poor route availability and thus results in an important data loss.

By adopting the concepts of Multihoming and hybrid network management, SCTP-WSN extension proves to be the best in terms of sparse WSN data transport, since it anticipates possible topology changes by creating a transparent data passage from an unavailable route to an available one. In addition, SCTP-WSN contributes to the optimization of power intake by providing necessary routing data to a sensor in order to establish alternative routes.

\section{CONCLUSION}

In order to guarantee the comprehension of the phenomena observed by sensor networks, the task of the latter is not only quantitative. It is rather qualitative. The limited radio capacity of the sensors makes it impossible for them to communicate unless with their direct neighbours. That is why routing protocols have to show more robustness when faced with instable topologies.

SCTP-WSN adopts the concept of Multihoming with the purpose of ensuring a fast and transparent broken-down route recovery, as well as providing a hybrid network management to facilitate the creation of alternative routes. However, the node Sink has to present physical and software performances that are commensurate with the size of the network.

To conclude, further work in this area should focus on developing new hybrid WSN managing architectures for the purpose of overcoming the shortcomings of WSN power intake and instable topologies.

\section{ACKNOWLEDGEMENT}

Special acknowledgement to SADOUNI Nesrine for her great contribution to the writing of this scientific paper.

\section{REFERENCES}

[1] Chi-Tsun Cheng, Member, IEEE, Chi K. Tse, Fellow, IEEE, and Francis C. M. Lau, Senior Member, IEEE (March 2011), "A Delay-Aware Data Collection Network Structurefor Wireless Sensor Networks", IEEE Sensors Journal, Vol. 11, No. 3.

[2] Chao Wang, Huadong Ma, Member, IEEE, Yuan He, Member, IEEE, and Shuguagn Xiong (June 2012), "Adaptive Approximate Data Collection for Wireless Sensor Networks", IEEE Transactions on Parallel and Distributed Systems, Vol 23, no.6, pp 1004-1016.

[3] Konstantopoulos, C.; Pantziou, G.; Gavalas, D.; Mpitziopoulos, A.; Mamalis, B. (May 2012), "A Rendezvous-Based Approach Enabling Energy-Efficient Sensory Data Collection with Mobile Sinks", IEEE Transactions on Parallel and Distributed Systems, vol.23, no.5, pp.809817.

[4] MEBARKI, A, BENABBAS, C., 2008, " Aménagement intégré des eaux du Kébir-Rhumel (Algérie Orientale) : le système Beni-Haroun », Communicationm, au "Colloque International Terre et Eau - 2008 », Université « Badji Mokhtar de Annaba »(Algérie), 17-19 novembre 2008

[5] Ankita M.Shendurkar, Nitin R.Chopde " A Review of Black Hole and Worm Hole Attack on AODV Routing Protocol in MANET" International Journal of Engineering Trends and Technology (IJETT) Volume 9 Number 8, 2014

[6] Kim K.,"A Cross Layered Approach for Multihoming on SCTP in mobile Ad hoc Networks", TENCON (Taipei International Convention Center), IEEE Region 10 Conference, 2007.

[7] Charoenpanyasak S.,Paillassa B.,"SCTP multihoming with Cross Layer Interface in Ad Hoc Multihomed Networks" IEEE Wi-Mob, New York USA, 2007.

[8] SADOUNI. S, Benslama. Malek, Beylot. A.L André-Luc2Stewart R "SCTP-MANET New Extension of SCTP Protocol for the Optimization of Manet Performances" International Journal of Wireless \& Mobile Networks (IJWMN) Vol. 8, No. 5, October 2016

[9] Y. Zaki, T. Weerawardane, C. Georg, and A. Timm-Giel, "Long Term Evolution (LTE) Model development within OPNET Simulation Environment," in OPNET Workshop 2011, Washington D.C., USA, Aug. 2011. 